\title{
A Fuzzy Logic-Based Approach for Humanized Driver Modelling
}

\author{
Yuxiang Feng $\mathbb{D}^{1},{ }^{1}$ Pejman Iravani $\mathbb{D},{ }^{2}$ and Chris Brace $\mathbb{D}^{3}$ \\ ${ }^{1}$ Department of Civil and Environmental Engineering, Imperial College London, London, UK \\ ${ }^{2}$ Department of Mechanical Engineering, University of Bath, Bath, UK \\ ${ }^{3}$ The Institute for Advanced Automotive Propulsion Systems, University of Bath, Bath, UK \\ Correspondence should be addressed to Yuxiang Feng; y.feng19@imperial.ac.uk
}

Received 11 April 2021; Accepted 16 June 2021; Published 23 June 2021

Academic Editor: Chi-Hua Chen

Copyright ( $\odot 2021$ Yuxiang Feng et al. This is an open access article distributed under the Creative Commons Attribution License, which permits unrestricted use, distribution, and reproduction in any medium, provided the original work is properly cited.

\begin{abstract}
All human drivers can be characterised by their habitual choice of driving behaviours, which results in a wide range of observed driving patterns and manoeuvres. Developing control strategies for autonomous vehicles that address this feature would increase the public acceptance of such vehicles. Therefore, this paper proposes a novel approach to developing rule-based fuzzy logic driver models that simulate different driving styles in the car-following regimes. These driver models were trained with the collected onroad driving data to capture corresponding human drivers' characteristics. The proposed approach consists of three main components: collecting on-road driving data, developing a vehicle model, and establishing the car-following driver models. Firstly, an instrumented vehicle was used to collect driving data over the same route for three consecutive months. Car-following scenarios during these journeys were extracted, and related data were processed accordingly. Afterwards, a representative model of the instrumented vehicle was created and evaluated. Finally, a fuzzy logic driver model that uses humanized inputs was developed and calibrated with the recorded data. The developed driver model's performance was assessed using the collected driving data and a baseline PID driver model. With the performance validated, models representing more aggressive and more defensive driving styles were derived following the same procedure. A cross-driver analysis was then implemented in a normalized car-following scenario with the established vehicle model to investigate the impacts of different driving styles further. The developed driver model can introduce driving styles into drive cycle experiments and replicate on-road real driving emission tests in the laboratory. Moreover, as the proposed method has high robustness to incomplete datasets, it can be a more cost-effective option to facilitate the development of humanized and customized vehicle control strategies for autonomous driving.
\end{abstract}

\section{Introduction}

Initially proposed by Elander et al. [1], the concept of driving style, or vaguely referred to as driver behaviour occasionally, can be defined as "driving style which concerns the way individuals choose to drive or driving habits that have become established over a period of years." While the consensus of a unified definition about this concept has not yet been reached [2-5], it generally refers to the driver's own habitual choice of driving manoeuvre, which can be reflected in both cognition and action characteristics of human drivers. The cognition aspect consists of the driver's evaluation of the current traffic scenario and the corresponding decision-making process. Meanwhile, the action aspect mainly relates to the driver's adopted driving patterns, such as the preference on the pattern of accelerating, braking, and gear shifting.

Similar to the vagueness in the definition of driving style, its exact classification can also vary significantly with the focus of research. For example, it can be as simple as two groups (aggressive and normal) [6,7] or categorized explicitly into eight groups (angry, high-velocity, risky, anxious, dissociative, patient, careful, and distress-reduction) [8]. Nonetheless, it should be noted that the most common trend in existing studies is to classify it into three distinct groups, namely, aggressive, normal, and defensive.

Aggressive driving style, or referred to as sporty, hostile, and angry driving style, is the behavioural pattern consisting of risky speeding, abrupt speed change, harsh acceleration and deceleration, and improper lateral position maintenance 
[9]. This type of driving style has received the most extensive research focus since it deviates from the norm and expected behaviour of a driver and can be a significant cause of increased fuel consumption and emissions and potentially even deadly crashes $[7,10]$. The second group of driving style, as indicated by its name, refers to the driving style that is most commonly witnessed which is neither too aggressive nor too defensive. Normal driving style often functions as a reference to isolate other driving styles, which can be used as the baseline for driving style classification. Defensive driving is often conceptualised as contrary to aggressive driving [11]. While it has not been specifically defined, defensive driving generally refers to moderate acceleration/deceleration, properly maintained headway distance, and careful traffic flow participation. It has a high correlation with normal driving but in a more passive manner.

Various studies have been conducted to facilitate the classification of driving styles. For example, methods based on fuzzy logic [12-14] and neural network $[6,15]$ were developed to directly differentiate driving styles from driving data. Other supervised [16] and unsupervised models $[17,18]$ have also been developed to facilitate driving style classification. Moreover, instead of using manoeuvre frequencies, Li et al. [19] categorized highway driving behaviours into 12 manoeuvre states and focused on the transition patterns with a random forest algorithm. It was found that a better driving style estimation can be achieved using the transition probabilities between manoeuvres.

While extensive studies have been conducted to investigate the influence of driving style on driving safety [20-22] and fuel consumption [23-25], they were mainly based on analysing human driving data collected under various scenarios. Although positive correlations between driving style and each factor were found within these studies, the exact influence of driving style difference is still under debate, especially for fuel consumption research. This is mainly caused by the unrepeatable nature of human behaviours, making comparative study challenging to implement. A viable solution is to develop a driver model that can mimic human behaviours and perform different driving styles to solve this issue.

Although the driving style difference can be reflected in various driving scenarios, such as car-following, free flow, and driving under instructions, car-following scenarios are preferred in this study for investigation. This is because carfollowing regimes are the most primary scenario encountered by human drivers nowadays [26]. Additionally, both cognition and action characteristics of driving style can be reflected in the driver's preferred headway distance and driving patterns in this regime. Therefore, this study aims to develop humanized driver models specifically in car-following regimes.

The major contribution of this paper is three-folded. First, a fuzzy logic-based humanized driver modelling approach was developed, which is capable of simulating different driving styles using environmental inputs from human driver perception. Second, an anchored procedure to the standard World-wide harmonized Light duty Test Cycle (WLTC) was proposed to facilitate the driver models' evaluation. Third, the difference in driving style and their influence on fuel consumption was evaluated in a unified comparative environment.

The remaining of this paper is structured into four sections. Section 2 briefly reviews the relevant literature, and Section 3 explains the adopted methodology. Afterwards, the obtained results and discussion of analysis are presented in Section 4. Finally, Section 5 summaries the findings and limitations of this study and suggests future studies' direction.

\section{Literature Review}

With the earliest research implemented back in the 1950s [27], various approaches have been adopted to develop carfollowing models, which can be roughly divided into two categories, namely, the explanatory and nonexplanatory models [28].

Explanatory models are mainly based on closed-form mathematical models and usually define an ego vehicle's movement as a function of headway distance, relative speed, and host speed. Existing studies adopting this approach include Tang et al. [29]. They incorporated individual preference on optimal speed and safe distance into the traditional full velocity difference (FVD) model as additional driver attributions. Another study based on FVD was implemented by Zhang et al. [30], which considered the acceleration of the preceding vehicle and the ego driver's driving style. Both studies have divided driving styles into three categories (aggressive, neutral, and conservative) and extracted features of each category to establish the respective driver models. Both studies found the developed models can effectively improve stability and realism than the conventional FVD models.

Model predictive control is another commonly adopted approach for developing explanatory models. For instance, Luo et al. [31] developed an MPC-based ACC algorithm that considered safety, comfort, fuel consumption, and car-following performance as additional constraints. Meanwhile, Zhang and Vahidi [32] investigated the adoption of ecodriving strategies and incorporated the prediction of the front vehicle's behaviour into their model, leading to the improved capability to effectively reduce fuel consumption.

These explanatory models' significant advantage is their computation efficiency, which enables them to be easily incorporated in traffic flow simulation, especially with a large scale of vehicles. Consequently, these models have been widely adopted in microscopic and macroscopic traffic simulation studies $[29,30]$. However, it should be noted that although the driving style parameters in some studies were calibrated from naturalistic driving data, these models' performance in mimicking human driving styles still lacks evaluation.

Unlike explanatory models defined using mathematical relations, nonexplanatory models are data driven, and the ego vehicle's movement is usually directly calibrated. Many data-driven models have been adopted in this research field. For example, Su et al. [33] categorized 84 drivers into three driving style groups and extracted features of each group to 
calibrate three representative driver models based on fuzzy logic. Meanwhile, another study based on fuzzy logic was conducted by Hao et al. [34]. They developed two generalised driving style models (aggressive and conservative) using selected vehicle trajectory data from a published dataset. A genetic algorithm was adopted for the calibration of the fuzzy membership function.

Artificial neural network (ANN) is another widely adopted approach for human driver modelling due to its performance in imitating human behaviours from the machine learning perspective. The earliest research can be traced back to 1998, when Macadam et al. [6] developed a car-following driver model based on a two-layer neural network. Although only one driver model was calibrated, it has achieved satisfying results in the preliminary evaluation, revealing the promising potential of developing driver models using neural networks. Bifulco et al. [35] developed three driver models based on ANN, linear function, and polynomial function. These models were calibrated and evaluated using the same dataset. While no significant difference was found among the three models, the threelayer feedforward neural network model showed more flexibility in learning capabilities. Moreover, a series of studies were conducted by Shi et al. [36-38], which developed driver models using different variations of ANN, e.g., cerebellar model articulation controller [36], radial basis function [37], and wavelet neural network [38]. The developed networks receive vehicle speed and speed change as input and generate throttle position and brake pressure as output. The personalised models were then employed to follow the FTP-75 drive cycle to normalize the driving style variations. While no comparisons were made among the adopted neural networks, their capabilities of capturing the behavioural characteristics of those drivers were confirmed.

Some modern machine learning approach has also been adopted in similar research. For instance, Wang et al. [39] trained a recurrent neural network (RNN) with gated recurrent units (GRUs), which yielded higher simulation accuracies than traditional models that only use instantaneous inputs. Meanwhile, Gao et al. [40] used inverse reinforcement learning to establish each driver's reward function and analysed their driving characteristics and car-following strategies. Jiang et al. [41] used maximum likelihood inverse reinforcement learning to estimate driving style parameters from the collected driving data. A longitudinal assistance strategy was then developed with the calibrated parameters based on inverse reinforcement learning. Zhu et al. [42] developed a deep deterministic policy car-following gradient model, which uses the disparity between simulated and observed speed as the reward function to reproduce car-following behaviours.

It can be noted that all these nonexplanatory models attempted to infer the inner relations between human driver's cognition and action directly from the naturalistic driving data, collected from either on-road instrumented vehicles or driving simulators. One significant advantage of these approaches over exploratory models is the improved performance in simulating humanized behaviours, as they are directly inferred from driving data. While these datadriven models' benefits are apparent, they also suffer from some drawbacks, most notably the expensive computation requirements and difficulty in interpretation. Owing to these algorithms' relatively more complex architecture, they have higher requirements on the hardware platform. Moreover, large amounts of naturalistic driving data are also required by these models for training and inferring. Therefore, these data-driven models are more commonly used in simulating microscopic traffic scenarios with a limited number of vehicles.

While these existing studies have achieved some success in modelling human driving styles, it should be noted that they mainly take the accurate measurements of the ego vehicle's speed and the speed difference between ego and the preceding vehicle as input to their models. As human drivers' perception of the traffic environment is based on estimations, these accurate measurements can impair the ability to capture cognition characteristics. Moreover, these variables may be inappropriate from human drivers' perception. Therefore, this study proposes to develop a fuzzy logic-based modelling approach, which uses more humanized inputs and can incorporate the vagueness in drivers' perception to improve the similarity to human reasoning. Moreover, it should be noted that fuzzy logic models also are more computationally efficient than other data-based models and are more robust to incomplete data and less accurate traffic environment measurements.

\section{Materials and Methods}

3.1. Naturalistic Driving Data Collection. To facilitate the naturalistic driving data collection, a VW Sharan was instrumented to record daily driving on a selected route (shown in Figure 1) for three consecutive months. The chosen route covers approximately 45 miles and consists of both urban and rural segments. During the data collection phase, a sum of 90 trips was recorded, with an average duration of 63 minutes. The recorded maximum vehicle speed during each trip was approximately $100 \mathrm{~km} / \mathrm{h}$.

The vehicle state information was directly retrieved from the OBD-II port using an Influx Rebel CT OBD data logger. Meanwhile, a continental $77 \mathrm{GHz}$ long-range ARS 308 radar and a Nextbase $512 \mathrm{G}$ dashcam were also instrumented on the vehicle to facilitate the collection of corresponding traffic information.

During the postdata processing, both the headway distance and the relative speed between the preceding and ego vehicles were extracted from the radar measurements. Simultaneously, the recorded dashcam footages were processed to generate a second source of headway distance measurements. Afterwards, a Kalman filter was developed to fuse these measurements and generate optimized headway distance estimation [43]. Synchronization between vehicle state information and traffic information was then implemented to form the naturalistic driving dataset for developing the driver model. 


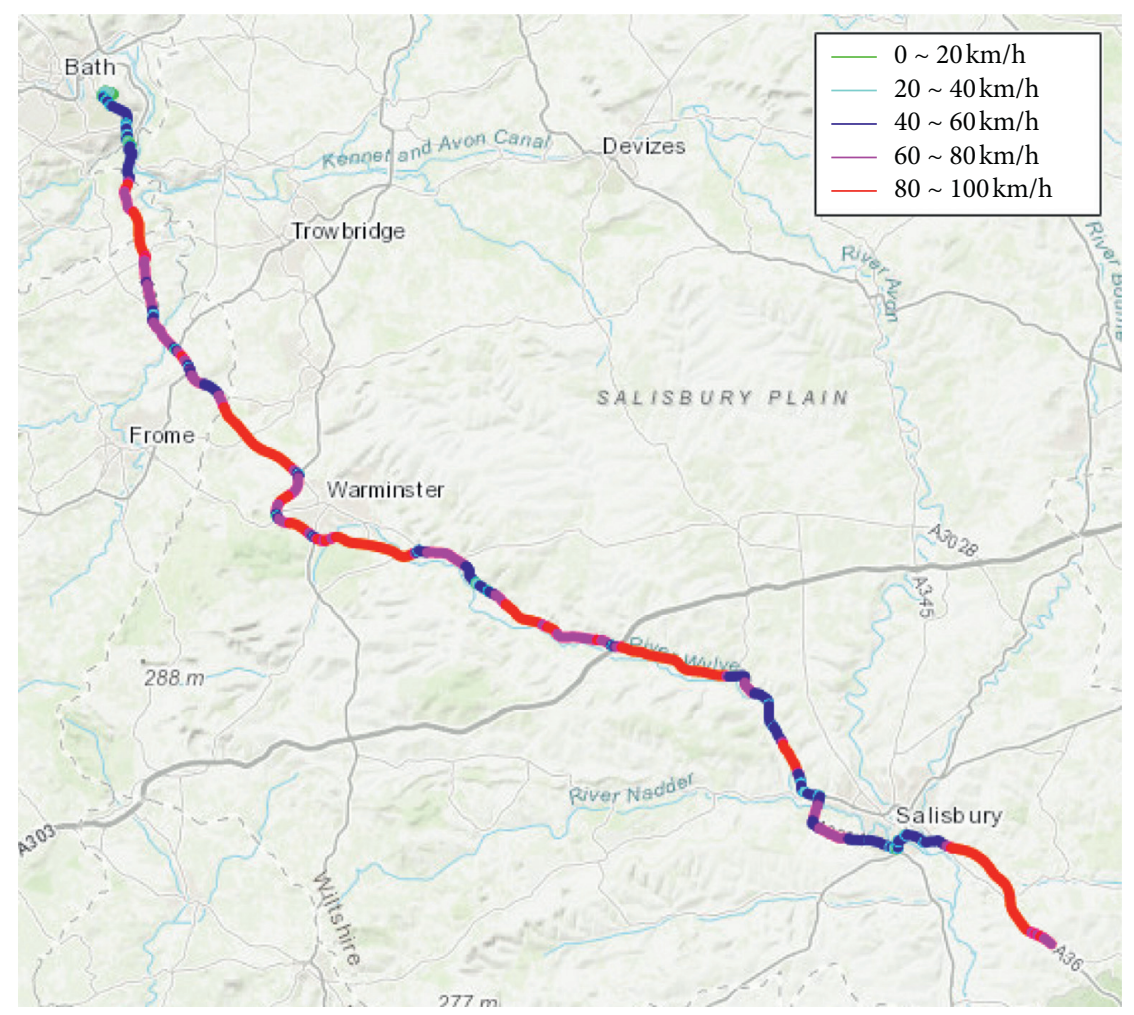

Figure 1: Route for driving data collection.

3.2. Vehicle Simulation Model. To facilitate the evaluation of the established driver model, a vehicle model that possesses the instrumented vehicle's essential features was developed. Using the vehicle parameters specified in [44], a corresponding vehicle model was developed in Simulink, a graphical programming environment for modelling, simulating, and analysing multidomain dynamical systems. It consists of five subsystems, namely, engine, brake, transmission, tire, and vehicle body. The architecture of the established vehicle model is illustrated in Figure 2.

3.3. Fuzzy Logic Driver Modelling. A fuzzy logic controller typically consists of five components: variables, rules, a fuzzifier, an inference engine, and a defuzzifier. Meanwhile, it also comprises four steps, initialization, fuzzification, inference, and defuzzification. Variables and rules are defined in the initialization phase. Variables are linguistic terms describing inputs and outputs, usually ranging from small to large. For each variable, it has a set of linguistic terms and associated membership functions.

Meanwhile, rules are the logic linkage between inputs and outputs and are used to determine correlating actions to each specific combination of inputs. Both parameters can be defined using the expert's knowledge during the initialization. After the initialization phase, the controller's actual inputs are transformed from crisp values to corresponding linguistic terms. Typically, two or more terms can be correlated with each input, and membership functions determine the degree of involvement. With the fuzzified inputs and corresponding rules, an inference engine can be used to compute each rule's contribution to the output. Finally, the accumulated output is transformed from fuzzy into crisp during the defuzzification phase.

With the general procedure of fuzzy logic outlined, it can be noted that fuzzy rules are the kernel of fuzzy logic and have a vast influence on its performance. Thus, these rules need to be appropriately defined to improve controller performance. To define such rules, the model's inputs and outputs need to be properly selected in advance. From drivers' perception perspective, multiple inputs can be received during driving, such as headway distance, relative speed to the preceding vehicle, current vehicle speed, engine rpm, gear selection, pedal position, road gradient, time, and weather. While all this information can influence drivers' decisions, it is unrealistic to consider all of them together, as the number of fuzzy rules will increase dramatically with multiple inputs. Thus, only the most prominent parameters, such as headway distance, current vehicle speed, gear selection, and pedal position, were selected to simplify the rule composition.

Meanwhile, two sets of headway distances were derived to improve the similarity to human drivers. While the actual distance measurement was used in a short range, the time gap measurement was adopted in middle and long ranges. This is mainly because of the widespread adoption of the two-second time gap rule in most countries. Drivers commonly obey it to assess safety headway distance by counting two seconds using stationary references. While this rule is more likely a reaction time-based guidance, it is useful when vehicle travels above a certain speed. A 


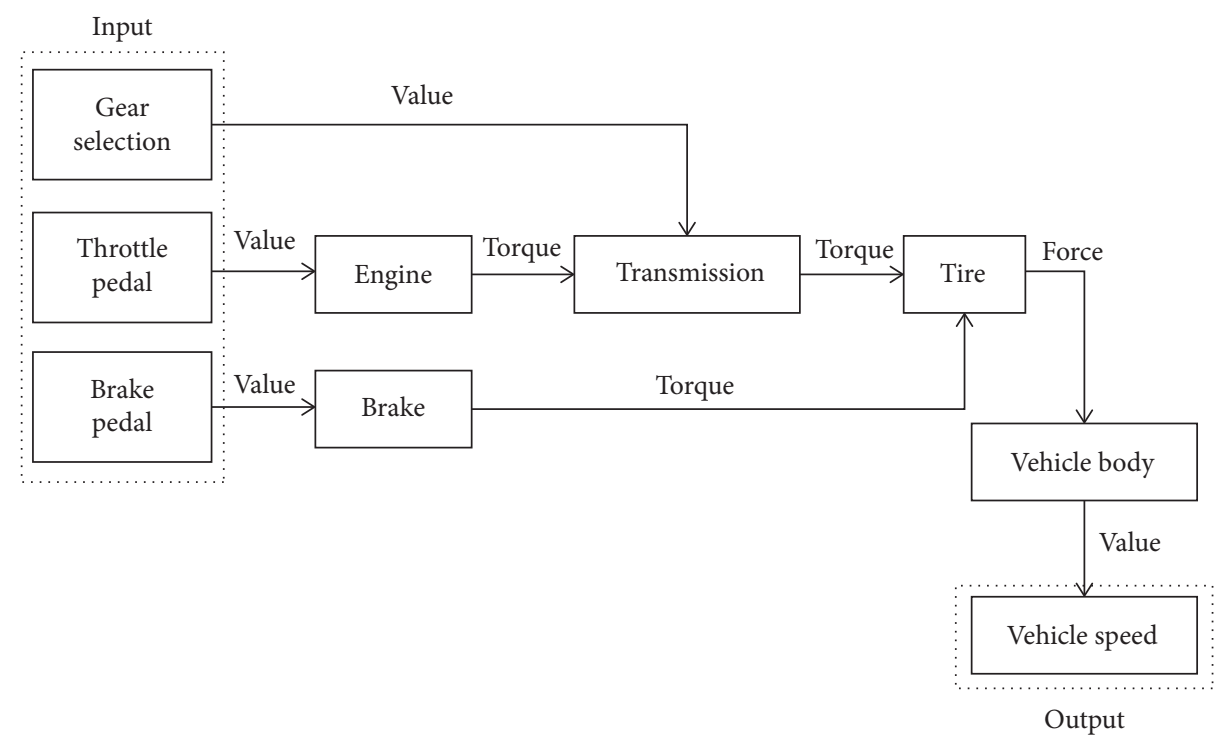

Figure 2: Architecture of the established vehicle model.

physical distance to a distant preceding vehicle can be challenging to estimate. However, this rule does not apply to slow-moving vehicles, and physical distance can be easily estimated instead. Therefore, both time and distance-based headway distances were used segmentally as model inputs, together with current vehicle speed, gear selection, and pedal position.

The typical driver outputs can be denoted as throttle pedal movement, brake pedal movement, and gear shifting. As throttle and brake pedals cannot be activated simultaneously and multiple outputs can increase fuzzy rules' complexity, power demand was selected as the sole output to simplify the fuzzy logic controller. A linear transformation was created between power demand and pedal movements. Thus, pedal commands can be derived accordingly from the output of the fuzzy logic controller. A gear shifting strategy based on vehicle speed was also developed.

According to experts' knowledge from the corresponding participant, five linguistic terms were derived for time gap (Very Small, Small, Middle, Large, and Very Large), current vehicle speed (Very Slow, Slow, Appropriate, Fast, and Very Fast), and power demand (Harsh Decelerate, Slight Decelerate, Maintain, Slight Accelerate, and Harsh Accelerate), respectively, and three for distance measurement (Close, Medium, and Far). Meanwhile, based on the number of variables, 15 rules were derived for the distance-based model and 25 rules for the time gap model. The control surface of each rule set is illustrated in Figure 3. The created rules share a similar structure, and one example can be described as follows: IF headway_distance is Close AND vehicle_speed is Fast THEN power_demand is Harsh Decelerate.

Meanwhile, Triangular-shaped membership functions were selected for each variable, which can be denoted as

$$
f(x)=\left\{\begin{array}{cc}
0 & x \leq a \\
\frac{x-a}{b-a} & a \leq x \leq b \\
\frac{c-x}{c-b} & b \leq x \leq c \\
0 & c \leq x
\end{array}\right\}
$$

where $a, b$, and $c$ are thresholds used to define the shape and location of the membership function.

As these thresholds have a vital influence on the established fuzzy controller's performance, they need to be correctly determined to optimize the controller. Therefore, these parameters should be calibrated using collected naturalistic driving data. There were 15 parameters associated with its membership function for a variable containing five linguistic terms and 9 for a variable with three linguistic terms. Carfollowing scenarios were isolated from the collected three months real driving data to calibrate these parameters. The recorded headway distance data and vehicle state information were synthesized and paired with the driver's intention, denoted by pedal movements and gear selection.

The method proposed by Yadav and Yadav [45] was adopted for the calibration of these parameters. According to their theory, each category of data was first sorted in ascending order, and then, $K$ means clustering was performed to cluster these values into separate groups. The number of clusters was determined by the quantity of linguistic terms for each variable. Meanwhile, the cluster centre value was assigned to $b$, the central vertex parameter. Moreover, the similarity value between adjacent data were computed as 


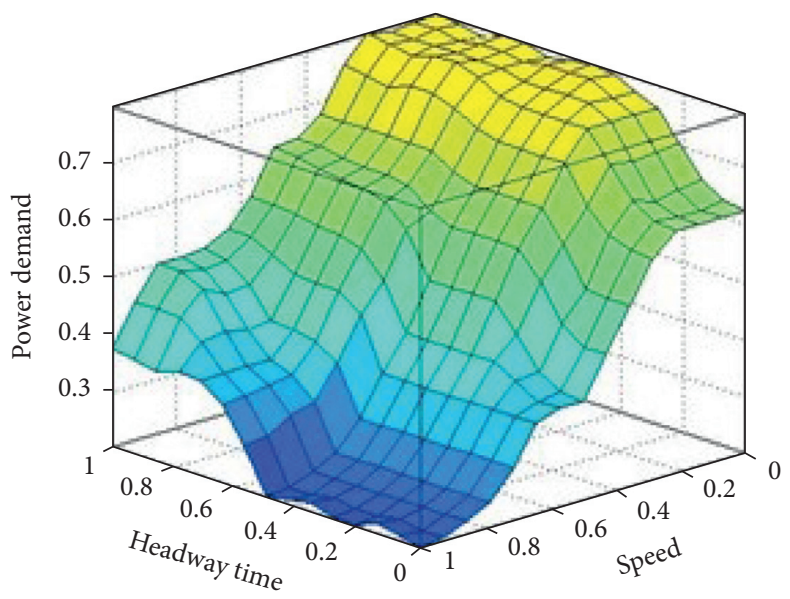

(a)

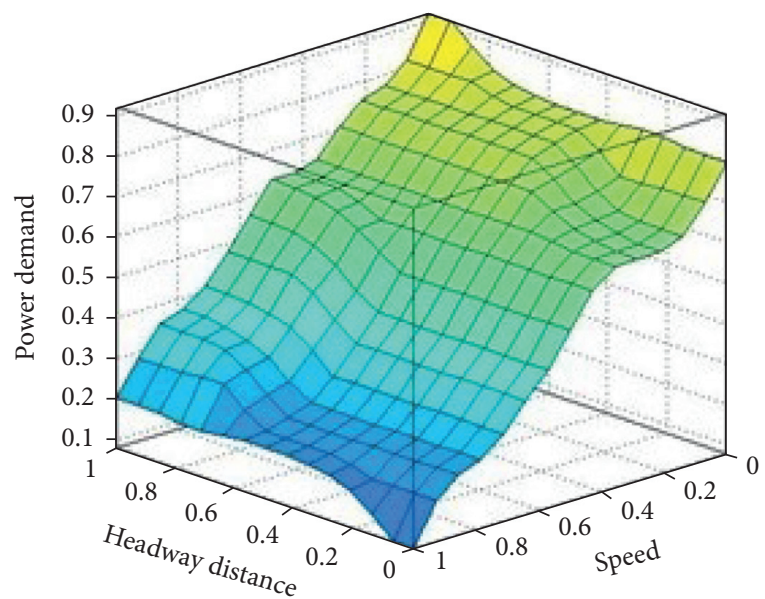

(b)

Figure 3: Control surface for each rule set. (a) Time based. (b) Distance based.

$$
S= \begin{cases}1-\frac{v_{i+1}-v_{i}}{C \times \sigma_{s}}, & \text { if } v_{i+1}-v_{i} \leq C \times \sigma_{s}, \\ 0, & \text { else, }\end{cases}
$$

where $S$ is the similarity, $v_{i}$ is the $i$ th data, $C$ is the control parameter, and $\sigma_{s}$ is the standard derivation of $v_{i+1}-v_{i}$.

Afterwards, each cluster's minimum value of similarity was selected as the membership value of two boundary points for that cluster. Therefore, the remaining two defining parameters can hence be computed as

$$
\begin{aligned}
& a=b-\frac{b-y_{\min }}{1-S_{\min }}, \\
& c=b+\frac{y_{\max }-b}{1-S_{\min }} .
\end{aligned}
$$

The obtained membership functions for each variable are illustrated in Figure 4.

To improve the simulation speed, this driver model was programmed as an $s$-function block. This setting can effectively reduce the consumed compiling time of the entire simulation model. Moreover, it can be noted that, for the two headway measurements, the test driver shows wider cognition of the short range, which indicates the tendency of maintaining medium or long headway distance. Meanwhile, the driver also favours driving at medium speed and has a wider recognition of very fast speed. As for the power demand, slight decelerate and slight accelerate cover the widest cognition zone. Thus, the driver prefers to change vehicle speed mildly and generally avoids harsh acceleration and deceleration. This calibration result indicated that the test driver possessed a normal to defensive driving style, coinciding with the driver's self-assessment and classification results [17].

3.4. Simulation Scenario. To facilitate the evaluation of the driver model's difference, a simulation scenario was developed to unify the comparison environment based on
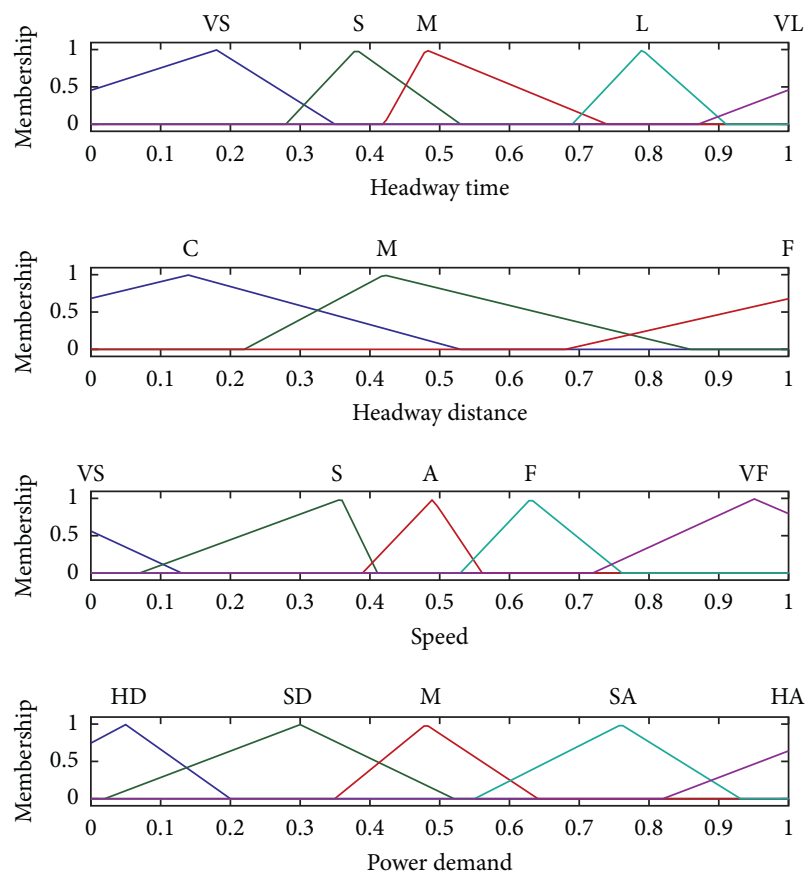

Figure 4: Calibrated membership functions.

WLTC, the latest drive cycle for emission test. Based on the power-weight ratio of the adopted instrumented vehicle, the Class 3 WLTC test cycle was selected [46], as illustrated in Figure 5.

To introduce driving style into this drive cycle data, one possible solution is assuming the driver is instructed to follow this speed profile. The driving style variance can then be partially reflected in the oscillations, convergent speed, and tracking accuracy. While these parameters can reveal the influence of driving style to a certain extent, they are not directly correlated, especially from the driver's perception. Therefore, an anchored procedure was proposed to convert this speed profile into a car-following scenario to incorporate the cognition characteristic of human drivers. A 


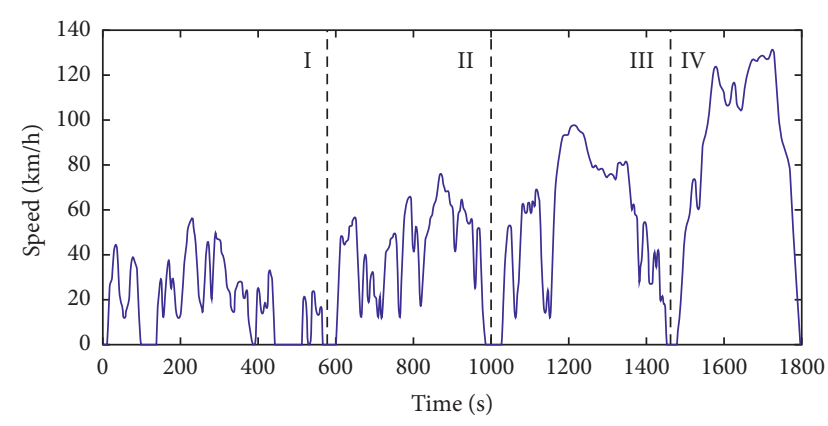

Figure 5: WLTC class 3 speed profile.

simulated preceding vehicle is introduced to create such a simulation scenario, which performs the WLTC speed profile. Thus, instead of directly providing the drive cycle data to the driver model, the drive cycle information is converted as the headway distance variations between the model and the simulated preceding vehicle. Hence, this setting can allow the driving style variances recorded in realworld car-following scenarios to be reflected in procedures that are anchored to standard drive cycle tests. While this setting can increase the difference between actual and target speed profiles, the similarity to human drivers is improved, and the influence of driving style can be more directly revealed.

Meanwhile, as shown in Figure 5, it can be noted that the WLTC drive cycle can be divided into four parts, namely, Low, Medium, High, and Extra High. Owing to the selected route, the majority of collected naturalistic driving data was within Low to High speed segments, which indicated that the calibrated driver model was also tuned explicitly for this speed range. Thus, the Extra High speed segment was discarded. Therefore, only the first three segments $(0-1460$ s) of the WLTC drive cycle were used in this study.

\section{Results and Discussion}

This section consists of four sections. The established vehicle model is evaluated first. Afterwards, an obtained driver model was compared with the corresponding human driver to examine the proposed modelling approach's performance. Moreover, two more driver models were developed to facilitate the investigation into the characteristics of different driving styles and their influence on fuel consumption. Finally, the limitation and direction for future work of this study is discussed.

4.1. Vehicle Model Validation. Before examining the driver model's performance, the established VW Sharan vehicle model should be validated as a simulation basis. As this study's overarching aim is to evaluate the driver model using the WLTC drive cycle, it should be worthy to first assess the vehicle model's performance with recorded actual inputs from the corresponding vehicle. Therefore, the 4WD AVL RoadSimTM 48" Chassis Dynamometer and the Stähle Autopilot SAP2000 robot driver within the Centre for Low Emission Vehicle Research of the University of Bath were used to acquire related experimental data. The Sharan was installed on the chassis dynamometer, and the robot driver was instructed to follow the WLTC Class 3 drive cycle speed profile. The vehicle state and robot driver information were recorded using a Rebel data logger. Essential information, such as vehicle speed, throttle pedal position, gear selection, engine rpm, robot accelerator leg, and brake leg movement, was extracted. While throttle pedal position, brake pedal position, and gear selection were required as vehicle model inputs, brake pedal position could not be directly recorded by the data logger. Therefore, a mitigation solution of obtaining brake pedal position was created for this validation. As the accelerator leg and brake leg movements of the Stäle robot were also recorded and the relation between pedal positions and leg movements should be fixed after installation, a mapping between vehicle pedal and robot leg was created using the throttle pedal and the accelerator leg. Afterwards, the brake pedal position can hence be obtained with this established mapping and record brake leg movement information. Both pedal positions and gear selection were transmitted to the vehicle model as inputs. The obtained vehicle speed profile is shown in Figure 6, together with the corresponding experimental result and computed error.

As illustrated in Figure 6, it can be noted that the simulation results generally possessed all the basic features of experimental data. As listed in Table 1, the total duration, accumulated stop duration, maximum and average speed of simulation, and experiment matched well between simulation and experiment.

4.2. Driver Model Examination. With the vehicle model validated, the established driver model's performance can hence be examined using the proposed simulation scenario. The speed profile of the first three segments of the WLTC drive cycle was assigned to the preceding vehicle. The total simulation time was set to 1460s. Meanwhile, an initial gap of $2 \mathrm{~m}$ was also assigned between the preceding and ego vehicle. Moreover, to increase the similarity to human drivers and boost simulation speed, a pedal change delay of $0.5 \mathrm{~s}$ was also introduced to the system to simulate human drivers' common cognitive delay time [47]. The calibrated normal driver model was examined first, and corresponding results are shown in Figure 7.

As shown in Figure $7(a)$, although the speed profile varies from the WLTC drive cycle, the basic features and tendencies are preserved. It can be noted that the driver model shows some oscillations, which were introduced by the $0.5 \mathrm{~s}$ pedal change delay. Meanwhile, the headway distance data illustrated in Figure 7(b) show that the driver model could perform a proper car-following behaviour, with the mean and maximum headway distance to be $12.50 \mathrm{~m}$ and $33.42 \mathrm{~m}$, respectively.

To evaluate the established fuzzy logic controller's performance in simulating human driving style, a traditional PID-based driver model was also developed and tested in the same simulation scenario. As the calibrated fuzzy logic driver model can be classified as a normal driver and 


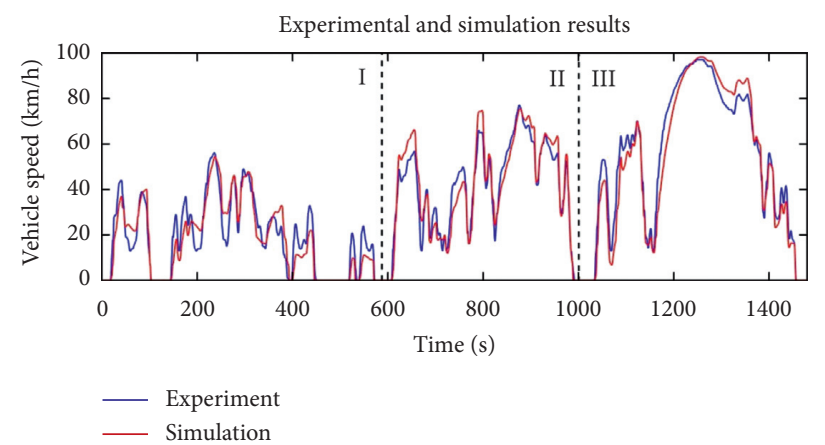

(a)

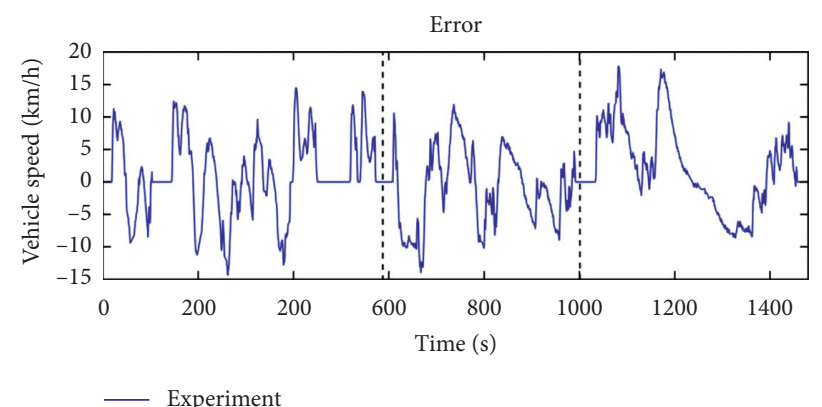

(b)

FIGURE 6: Speed comparison between simulation and experiment.

TABle 1: Comparison between simulation and experiment.

\begin{tabular}{|c|c|c|c|c|c|c|}
\hline \multirow{2}{*}{ Category } & \multicolumn{3}{|c|}{ Experiment } & \multicolumn{3}{|c|}{ Simulation } \\
\hline & I & II & III & I & II & III \\
\hline Duration (s) & 573 & 418 & 466 & 571 & 418 & 467 \\
\hline Stop duration (s) & 140 & 35 & 42 & 145 & 37 & 45 \\
\hline Max speed $(\mathrm{km} / \mathrm{h})$ & 56 & 77 & 97 & 54 & 76 & 97 \\
\hline Average speed $(\mathrm{km} / \mathrm{h})$ & 19.2 & 40.1 & 60.0 & 23.1 & 39.4 & 57.2 \\
\hline
\end{tabular}

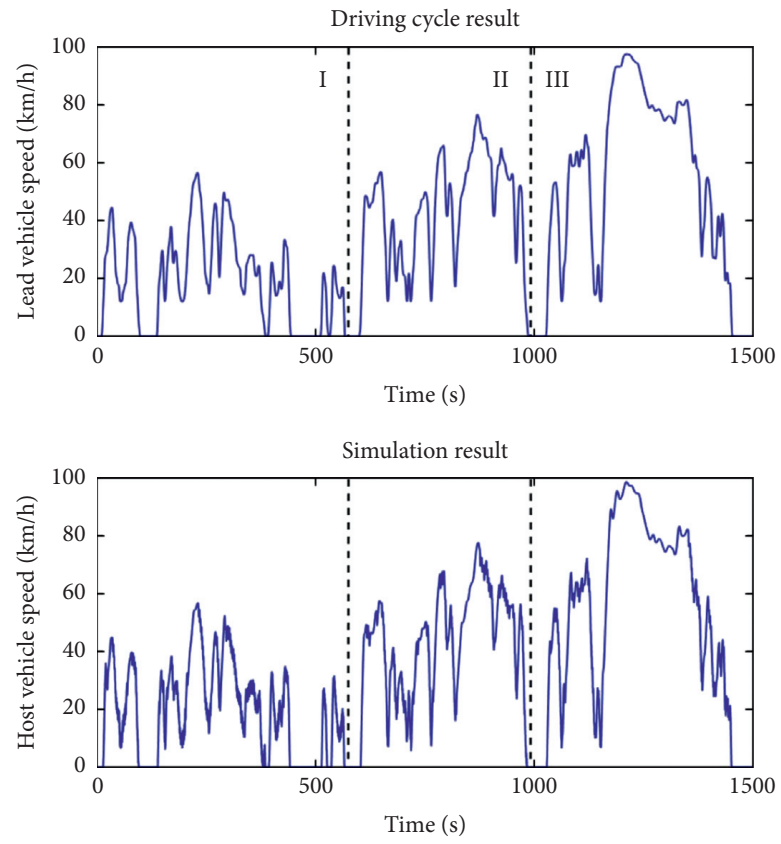

(a)

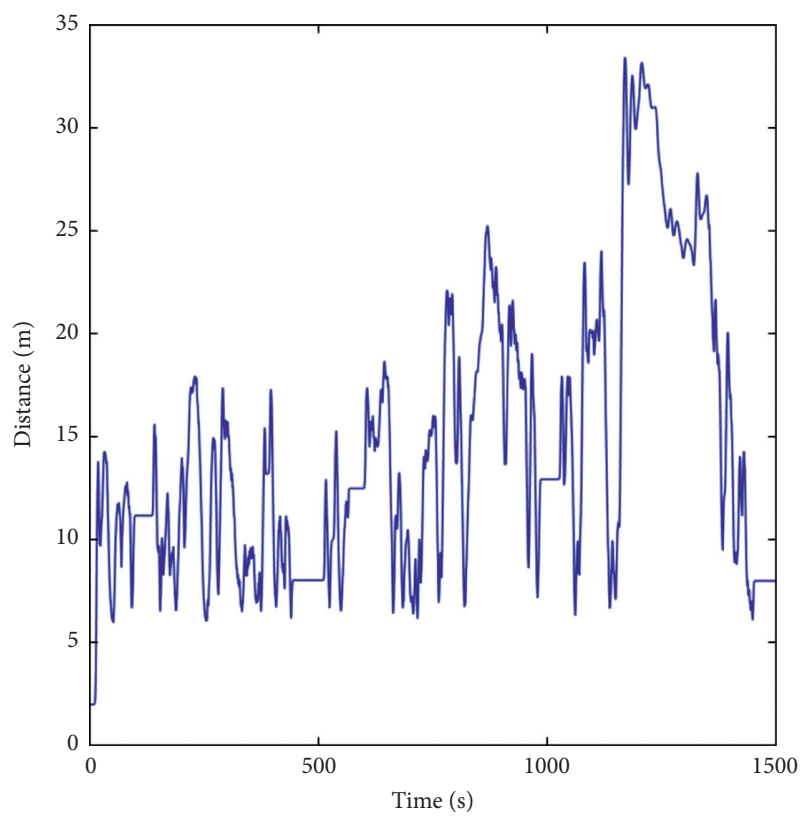

(b)

FIgURE 7: Simulation results of normal driver model. (a) Speed. (b) Headway distance.

generally maintain the 2 s time gap, the PID driver model was designed to also hew to the same time gap and follow a leading vehicle performing WLTC drive cycle. As a direct measure of driver's characteristic, throttle pedal demand and time gap were selected to compare the human driver and two simulation models. Meanwhile, both variables for the human driver were averaged from all naturalistic driving data to reduce any potential basis in a single trip. The obtained probability density distribution of throttle pedal position and time gap are illustrated in Figure 8.

As shown in Figure 8(a), it can be noted that the variations of throttle pedal distribution between the human driver and fuzzy controller are much smaller than the difference between the human driver and PID controller. The PID controller possessed a higher proportion of throttle value between $70 \%$ and $100 \%$, which indicated that 


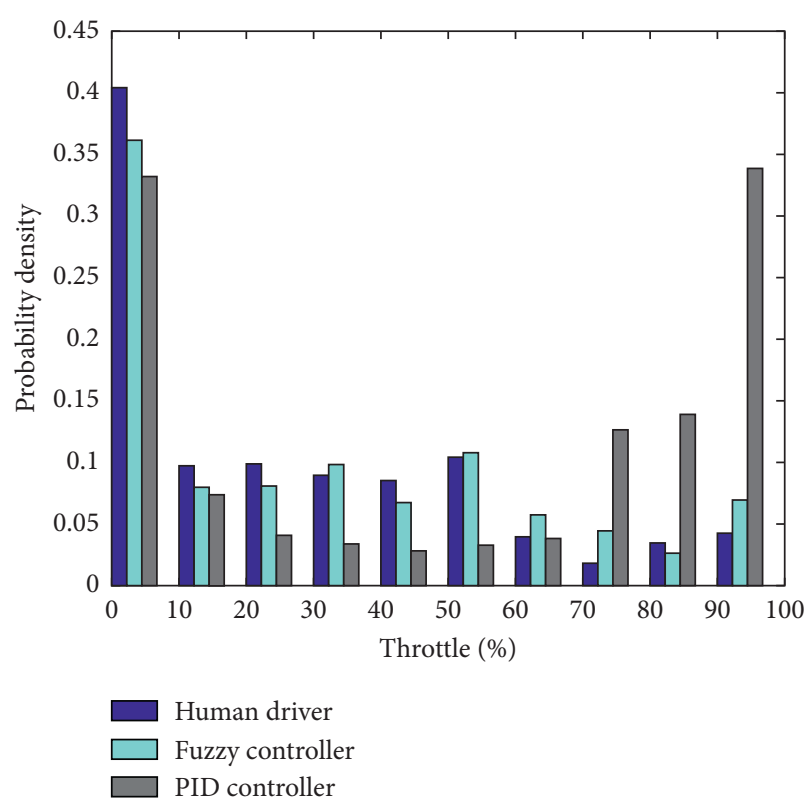

(a)

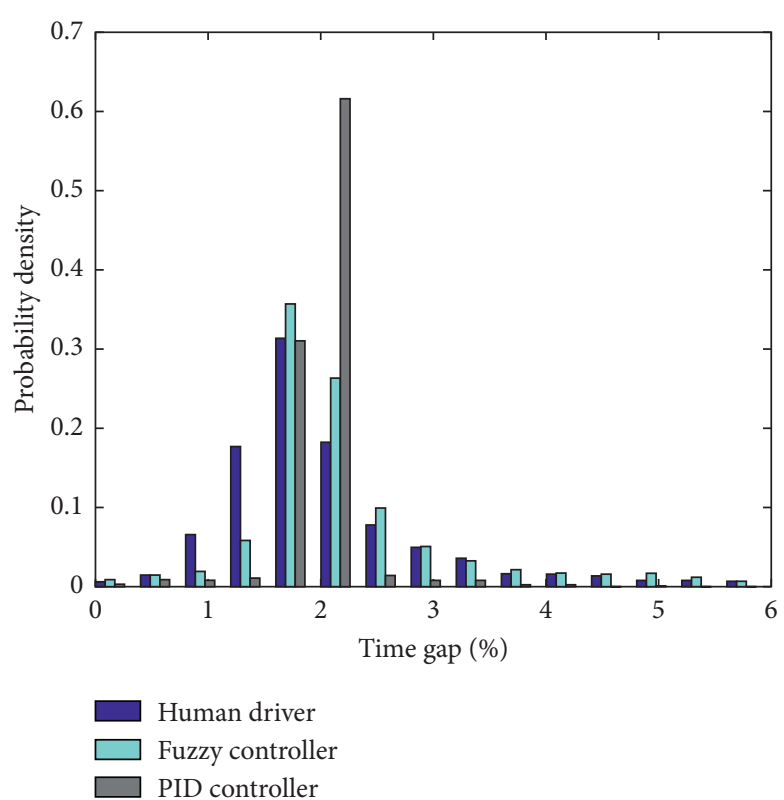

(b)

Figure 8: Driver model examination. (a) Throttle pedal. (b) Time gap.

the PID controller accelerated harsher to maintain the required time gap. Although the human driver had a larger proportion for the smaller throttle value, this may be mainly caused by complex traffic conditions and repeated stop-start scenarios. Aside from this segment, the probability distribution of the human driver and the fuzzy controller between $10 \%$ and $100 \%$ was similar, with the largest difference being 0.05 , which occurred between $20 \%$ and $30 \%$. From the distribution of probability density of throttle demands, it can be noted that the established fuzzy logic controller shows a better performance in simulating the human driver. Moreover, the time gap distribution was also extracted and illustrated in Figure 8(b). The intervals were increased to $0.4 \mathrm{~s}$ for better visualization. It can be noted that the human driver shows a larger proportion of smaller time gaps, which is similar to the throttle pedal distribution and could be the consequence of complex traffic scenarios. Although there were some variations between the fuzzy controller and human driver's time gap distribution, the established fuzzy controller still outperformed the PID controller.

4.3. Driving Style Comparison. To facilitate the investigation into the difference of driving style, the same procedure was repeated to develop two additional driver models. Following the driving style classification results in [17], these two human participants possess a relatively more aggressive and defensive driving style, respectively. The obtained membership functions of these two driver models are illustrated in Figure 9.

According to Meiring and Myburgh [9], aggressive driving style, or referred to as sporty, hostile, or angry driving style occasionally, is a behavioural pattern containing risky speeding, abrupt speed change, harsh acceleration and deceleration, and improper lateral position maintenance. Meanwhile, defensive driving is defined as contrary to aggressive driving, which generally refers to moderate acceleration and deceleration, wellmaintained headway distance, and careful participation in the traffic flow [48]. It has a high correlation with normal driving with a more passive manner. It can be noted from Figure 9 that the aggressive driver favours smaller safety distances and faster car-following speed, while the defensive driver possesses the opposite trend. For example, when headway time is 0.5 , it will be perceived by the aggressive driver as a combination of "Middle" and "Large" and defensive driver as "Small" and "Middle," which reflects the cognitive difference of these driving styles. Both calibrated driver models were also examined in the proposed simulation scenario to evaluate these driving styles and investigate their influence on fuel consumption.

As shown in Figure 10(a), the headway distances vary among these three styles. It was found that the mean and maximum headway gaps were $6.76 \mathrm{~m}$ and $23.52 \mathrm{~m}$ for the aggressive model, $12.50 \mathrm{~m}$ and $33.42 \mathrm{~m}$ for the normal model, and $17.94 \mathrm{~m}$ and $52.46 \mathrm{~m}$ for the defensive model. Thus, it can be noted that the aggressive model showed a greater tendency of tailgating, while the defensive model remained the largest safe distance. Moreover, to further evaluate this tendency, the headway distance distributions of each driver model were computed. It was found that the aggressive model is more distributed $(82.5 \%)$ in the small headway distance zone $(0-10 \mathrm{~m})$, while the defensive model occupies the largest proportion $(76.9 \%)$ for a headway gap larger than $15 \mathrm{~m}$. This revealed variation of headway distance is in coincidence with the common definitions of these three driving styles, as the headway gap is a crucial measure of aggressivity. 

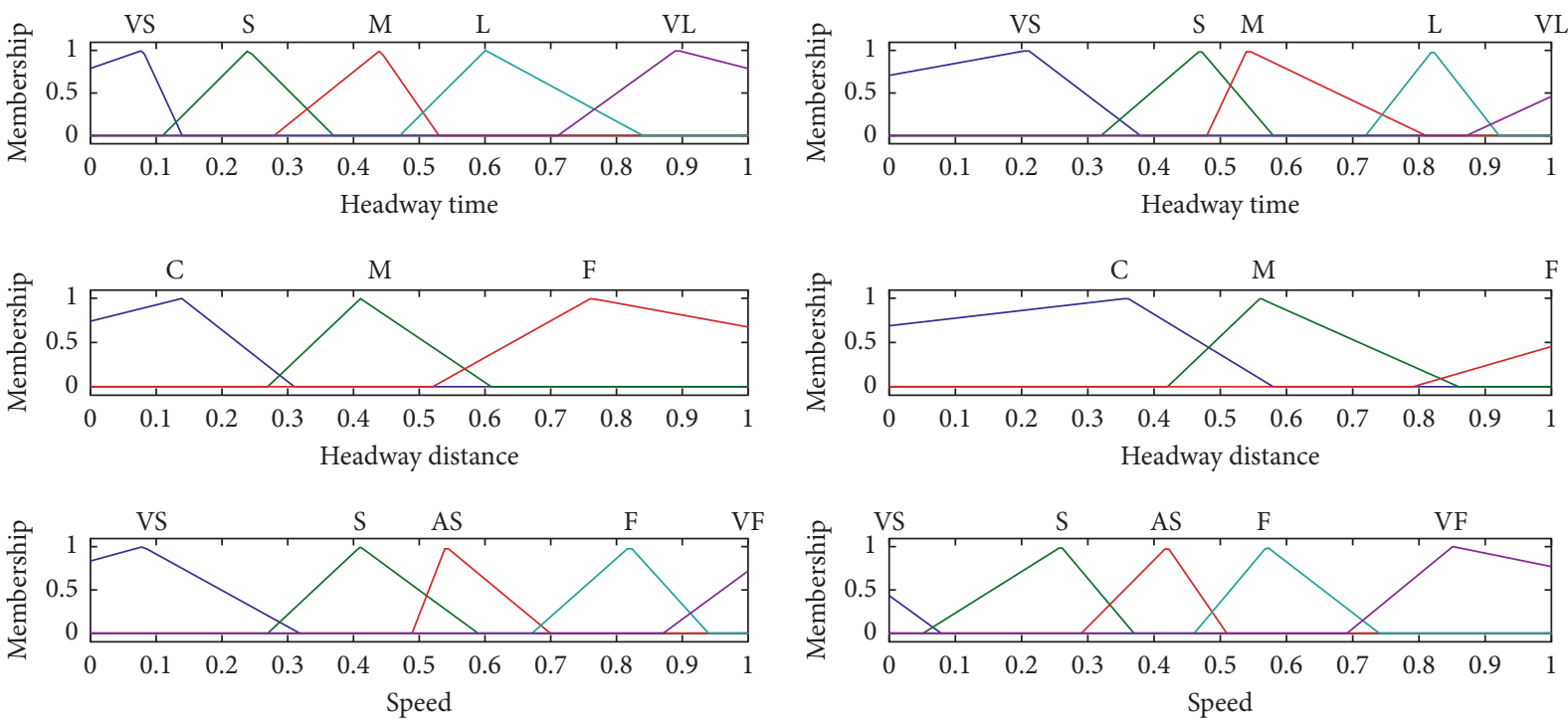

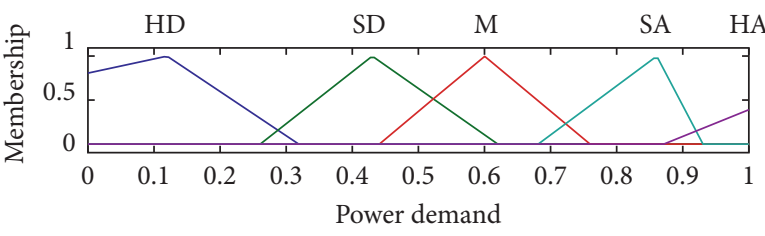

(a)

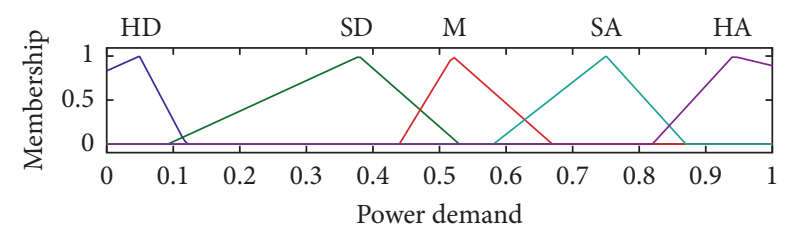

(b)

Figure 9: Calibrated membership functions. (a) Aggressive model. (b) Defensive model.
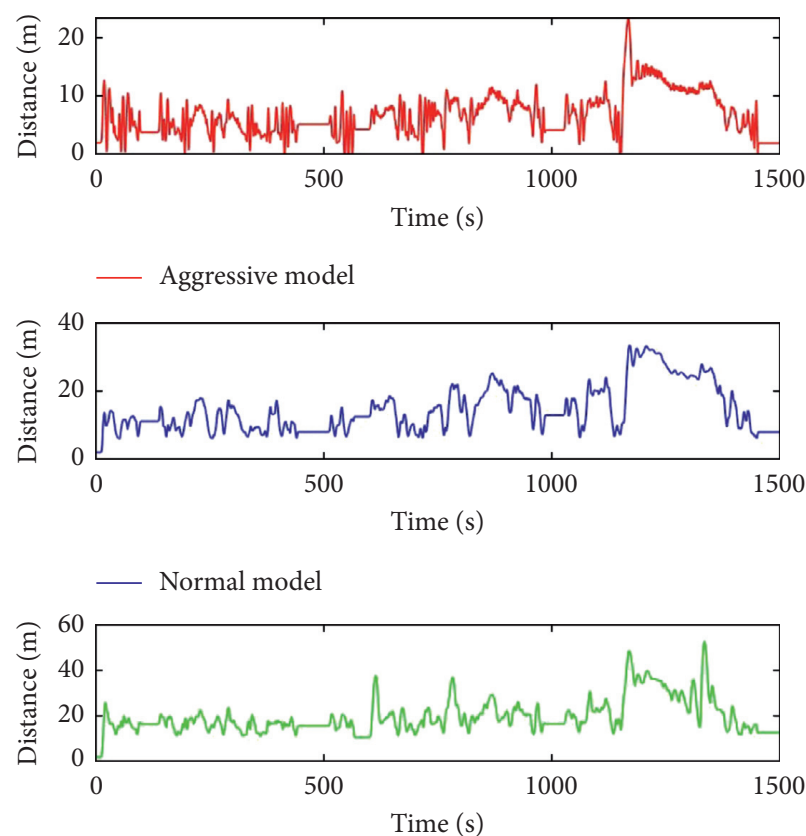

_ Defensive model

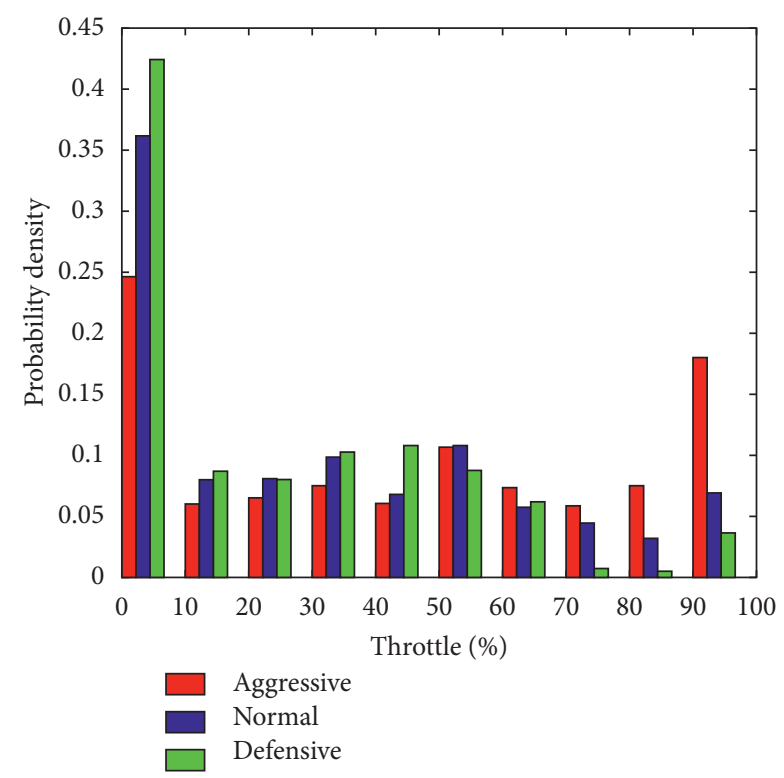

(a)

(b)

Figure 10: Driving style comparison. (a) Headway distance. (b) Throttle pedal demand. 
Meanwhile, the probability density distributions of throttle pedal demands were also selected to investigate the difference between the three driving styles, as it can directly reveal the driver's intention. From Figure 10(b), it can be noted that the defensive model possesses a larger proportion of smaller throttle demands, while the aggressive model shows a greater tendency of harsh acceleration. Meanwhile, the calibrated normal model's probability density was almost equally distributed, with a smaller proportion between $70 \%$ and $90 \%$. Although the changing tendencies of probability density among the three driving styles were not consistent within some throttle segments, it can be noted that the aggressive driving shows a preference for larger throttle values and hence harsher accelerations. Moreover, as jerks were also widely used for driving style classification [49], the average jerks of these three models were hence computed using the corresponding speed profiles to further validate these three relative driving style models, and they were normalized using the jerk of the WLTC drive cycle. The normalized jerk values for aggressive, normal, and defensive models were $2.1343,1.2237$, and 0.9046 , respectively. As a larger jerk denotes harsher accelerations and decelerations, the obtained results also demonstrated that three relative driving styles' modelling was successful.

The accumulated fuel consumption of the three models was also estimated during the simulation. It was found that the difference between the established driving style models in the Low speed segment is not prominent. This could be caused by the relatively small following distance, as aggressive driving is restricted in this scenario. Meanwhile, the variations of fuel consumption become more significant in the Medium and High speed segments, revealing the potential influence of driving style on fuel consumption. The estimated total fuel consumption of the aggressive driver model was about 1.16 litre, which was approximately $6 \%$ more when compared with the defensive driver model.

4.4. Limitation and Future Work. While the proposed method has achieved promising performance in simulating humanized driving style, some limitations are identified. It should be noted that although the proposed method shows higher robustness to incomplete data during the experiment, its performance can be affected by the suitability of expert knowledge. Therefore, the proposed modelling method can perform better when accurate expert knowledge can be obtained from the corresponding human driver.

As the future work, more environmental inputs, such as road gradient, time, and weather, can be incorporated to maximize the similarity to human drivers. Moreover, different test drivers' naturalistic driving data will be collected and used to formulate more accurate driving style variance. Furthermore, other calibration approaches will also be employed to improve the fuzzy logic controller's calibration process. Humanized driver models trained using the proposed approach can also be integrated with the decisionmaking process when designing advanced driver assistance system (ADAS) and the control strategy of autonomous vehicles (AVs) [50].

\section{Conclusion}

The primary aim of evaluating different driving styles by developing respective driver models was achieved. A datadriven approach to develop the humanized driver model was developed. A VW Sharan was instrumented to facilitate the data collection. The vehicle state information was logged using a Rebel data logger, and headway distance was obtained from a continental radar and a Nextbase dashcam. Aside from the naturalistic driving data collection, the simulation was entirely based on Simulink. A vehicle model denoting VW Sharan was first developed and validated using WLTC drive cycle data collected from chassis dynamometer experiments. Afterwards, a fuzzy logic driver model was established, and the membership functions were calibrated using naturalistic driving data. It was found that the established driver model was capable of simulating three relative driving styles. A PID-based driver model was also developed for comparison. Those driver models created with the proposed approach were found to perform better in preserving the driving style of corresponding human drivers. The influence of driving styles on fuel consumption was also compared with the obtained models. It was found that the aggressive model can consume approximately $6 \%$ more fuel than the defensive model.

This study's major contribution was to theoretically propose a novel approach for humanized driver modelling, which is capable of simulating different driving styles using environmental inputs from human driver perception. Moreover, an anchored procedure to standard drive cycle test was proposed, which can incorporate the driving style difference into these tests. Furthermore, the difference in driving style and their influence on fuel consumption was evaluated in a unified comparative environment.

\section{Data Availability}

The data used to support the findings of this study are available from the corresponding author upon request.

\section{Conflicts of Interest}

The authors declare that they have no conflicts of interest.

\section{References}

[1] J. Elander, R. West, and D. French, "Behavioral correlates of individual differences in road-traffic crash risk: an examination of methods and findings," Psychological Bulletin, vol. 113, no. 2, pp. 279-294, 1993.

[2] F. Saad, "Behavioural adaptations to new driver support systems: some critical issues," in Proceedings of the 2004 IEEE International Conference on Systems, Man and Cybernetics, pp. 288-293, The Hague, Netherlands, October 2004.

[3] H. A. Deery, "Hazard and risk perception among young novice drivers," Journal of Safety Research, vol. 30, no. 4, pp. 225-236, 1999.

[4] T. Lajunen and T. Özkan, "Self-report instruments and methods," in Handbook of Traffic Psychology, B. Porter, Ed., Elsevier, Amsterdam, Netherlands, 2011. 
[5] L. M. B. Kleisen, The relationship between thinking and driving styles and their contribution to young driver road safety, Dissertation (PhD), University of Canberra, Canberra, Australia, 2011.

[6] C. Macadam, Z. Bareket, P. Fancher, and R. Ervin, "Using neural networks to identify driving style and headway control behavior of drivers," Vehicle System Dynamics, vol. 29, no. 1, pp. 143-160, 1998.

[7] D. A. Johnson and M. M. Trivedi, "Driving style recognition using a smartphone as a sensor platform," in Proceedings of the 14th International IEEE Conference on Intelligent Transportation Systems, pp. 1609-1615, Washington DC, USA, October 2011.

[8] O. Taubman-Ben-Ari, M. Mikulincer, and O. Gillath, "The multidimensional driving style inventory-scale construct and validation," Accident Analysis \& Prevention, vol. 36, no. 3, pp. 323-332, 2004.

[9] G. Meiring and H. Myburgh, "A review of intelligent driving style analysis systems and related artificial intelligence algorithms," Sensors, vol. 15, no. 12, pp. 30653-30682, 2015.

[10] H. Y. Zhao, H. Zhou, C. F. Chen, and J. M. Chen, "Join driving: a smart phone-based driving behaviour evaluation system," in Proceedings of the 2013 IEEE Global Communications Conference, pp. 48-53, Atlanta, GA, USA, December 2013.

[11] F. Sagberg, S. Selpi, G. F. Bianchi Piccinini, and J. Engström, "A review of research on driving styles and road safety," Human Factors: The Journal of the Human Factors and Ergonomics Society, vol. 57, no. 7, pp. 1248-1275, 2015.

[12] A. Aljaafreh, N. Alshabatat, and M. S. N. Al-Din, "Driving style recognition using fuzzy logic," in Proceedings of the 2012 IEEE International Conference on Vehicular Electronics and Safety, pp. 460-463, Istanbul, Turkey, July 2012.

[13] M. S. N. Al-Din, A. Aljaafreh, N. Albdour, and M. Saleh, "Driving styles recognition using decomposed fuzzy logic system," International Journal of Electrical, Electronics \& Computer Systems, vol. 16, no. 1, pp. 1-5, 2013.

[14] D. Dörr, D. Grabengiesser, and F. Gauterin, "Online driving style recognition using fuzzy logic," in Proceedings of the 17th International IEEE Conference on Intelligent Transportation Systems, pp. 1021-1026, Qingdao, China, October 2014.

[15] J. E. Meseguer, C. T. Calafate, J. C. Cano, and P. Manzoni, "Characterizing the driving style behaviour using artificial intelligence techniques," in Proceedings of the 38th IEEE Conference On Local Computer Networks, pp. 1-3, Sydney, Australia, October 2013.

[16] W. S. Wang and J. Q. Xi, “A rapid pattern-recognition method for driving styles using clustering-based support vector machines," in Proceedings of the 2016 American Control Conference, pp. 5270-5275, Boston, MA, USA, July 2016.

[17] Y. Feng, S. Pickering, S. Pickering, E. Chappell, P. Iravani, and C. Brace, "A support vector clustering based approach for driving style classification," International Journal of Machine Learning and Computing, vol. 9, no. 3, pp. 344-350, 2019.

[18] G. F. Li, Y. Y. Chen, D. P. Cao et al., "Extraction of descriptive driving patterns from driving data using unsupervised algorithms," Mechanical Systems and Signal Processing, vol. 156, Article ID 107589, 2021.

[19] G. Li, S. E. Li, B. Cheng, and P. Green, "Estimation of driving style in naturalistic highway traffic using maneuver transition probabilities," Transportation Research Part C: Emerging Technologies, vol. 74, pp. 113-125, 2017.
[20] J. L. Deffenbacher, E. R. Oetting, and R. S. Lynch, "Development of a driving anger scale," Psychological Reports, vol. 74, no. 1, pp. 83-91, 1994.

[21] J. C. F. de Winter and D. Dodou, "The driver behaviour questionnaire as a predictor of accidents: a meta-analysis," Journal of Safety Research, vol. 41, no. 6, pp. 463-470, 2010.

[22] S. Amado, E. Ankan, G. Kaca, M. Koyuncu, and B. N. Turkan, "How accurately do drivers evaluate their own driving behavior? an on-road observational study," Accident Analysis \& Prevention, vol. 63, pp. 65-73, 2013.

[23] J. Tulusan, T. Staake, and E. Fleisch, "Providing eco-driving feedback to corporate car drivers: what impact does a smartphone application have on their fuel efficiency?" in Proceedings of the 14th ACM International Conference on Ubiquitous Computing, pp. 212-215, Pittsburgh, PA, USA, September 2012.

[24] M. Staubach, N. Schebitz, F. Köster, and D. Kuck, "Evaluation of an eco-driving support system," Transportation Research Part F: Traffic Psychology and Behaviour, vol. 27, pp. 11-21, 2014.

[25] M. J. M. Sullman, L. Dorn, and P. Niemi, "Eco-driving training of professional bus drivers - does it work?" Transportation Research Part C: Emerging Technologies, vol. 58, pp. 749-759, 2015.

[26] H. Liu, F. Sun, D. Guo, B. Fang, and Z. Peng, "Structured output-associated dictionary learning for haptic understanding," IEEE Transactions on Systems, Man, and Cybernetics: Systems, vol. 47, no. 7, pp. 1564-1574, 2017.

[27] W. A. Tillmann and G. E. Hobbs, "The accident-prone automobile driver," American Journal of Psychiatry, vol. 106, no. 5, pp. 321-331, 1949.

[28] H. B. Gao, G. Y. Shi, G. T. Xie, and B. Cheng, "Car-following method based on inverse reinforcement learning for autonomous vehicle decision-marking," International Journal of Advanced Robotic Systems, vol. 16, no. 6, pp. 1-11, 2018.

[29] T.-Q. Tang, J. He, S.-C. Yang, and H.-Y. Shang, "A car-following model accounting for the driver's attribution," Physica A: Statistical Mechanics and Its Applications, vol. 413, pp. 583-591, 2014.

[30] Y. Zhang, P. Ni, M. Li, H. Liu, and B. Yin, "A new car-following model considering driving characteristics and preceding vehicle's acceleration," Journal of Advanced Transportation, vol. 2017, pp. 1-14, 2017.

[31] L.-H. Luo, H. Liu, P. Li, and H. Wang, "Model predictive control for adaptive cruise control with multi-objectives: comfort, fuel-economy, safety and car-following," Journal of Zhejiang University Science A, vol. 11, no. 3, pp. 191-201, 2010.

[32] C. Zhang and A. Vahidi, "Predictive cruise control with probabilistic constraints for eco driving," in Proceedings of the ASME 2011 Dynamic Systems and Control Conference, pp. 1-6, Arlington, TX, USA, November 2011.

[33] C. Su, W. W. Deng, R. He, J. Wu, and Y. D. Jiang, "Personalised adaptive cruise control considering drivers' characteristics," 2018.

[34] H. Hao, W. Ma, and H. Xu, "A fuzzy logic-based multi-agent car-following model," Transportation Research Part C: Emerging Technologies, vol. 69, pp. 477-496, 2016.

[35] G. N. Bifulco, F. Simonelli, and R. D. Pace, "Experiments toward an human-like adaptive cruise control," in Proceedings of the 2008 IEEE Intelligent Vehicles Symposium, pp. 919-924, Eindhoven, Netherlands, June 2008.

[36] B. Shi, W. Meng, H. Liu, J. Hu, and L. Xu, “A normalized approach for evaluating driving styles based on personalized driver modeling," Lecture Notes in Electrical Engineering, in 
Proceedings of the 2014 SAE-China Congress \& China Automotive Engineering and Manufacturing Expo, pp. 433-444, Shanghai, China, October 2014.

[37] B. Shi, L. Xu, J. Hu et al., "Evaluating driving styles by normalizing driving behavior based on personalized driver modeling," IEEE Transactions on Systems, Man, and Cybernetics: Systems, vol. 45, no. 12, pp. 1502-1508, 2015.

[38] B. Shi, L. Xu, H. Jiang, and W. Meng, "Comparing fuel consumption based on normalised driving behaviour: a case study on major cities in China," IET Intelligent Transport Systems, vol. 11, no. 4, pp. 189-195, 2017.

[39] X. Wang, R. Jiang, L. Li, Y. Lin, X. Zheng, and F. Y. Wang, "Capturing car-following behaviors by deep learning," IEEE Transactions on Intelligent Transportation Systems, vol. 19, no. 3, pp. 910-920, 2017.

[40] H. Gao, G. Shi, G. Xie, and B. Cheng, "Car-following method based on inverse reinforcement learning for autonomous vehicle decision-making," International Journal of Advanced Robotic Systems, vol. 15, no. 6, pp. 1-11, 2018.

[41] Y. D. Jiang, W. W. Deng, J. S. Wang, and B. Zhu, Studies on Drivers' Driving Styles Based on Inverse Reinforcement Learning, SAE Internationals, Warrendale PA, USA, 2018.

[42] M. Zhu, X. Wang, and Y. Wang, "Human-like autonomous car-following model with deep reinforcement learning," Transportation Research Part C: Emerging Technologies, vol. 97, pp. 348-368, 2018.

[43] Y. X. Feng, S. Pickering, E. Chappell, P. Iravani, and C. Brace, "Distance estimation by fusing radar and monocular camera with kalman filter," 2017.

[44] P. Volkswagen Sharan (2010 onwards) Specs \& Dimensions [Online]. Peterborough: Parkers. Available from: http:// www.parkers.co.uk/volkswagen/sharan/estate-2010/20-tdicr-bluemotion-tech-(140bhp)-s-5d/specs.

[45] H. B. Yadav and D. K. Yadav, "A method for generating membership function from numerical data," Journal of Intelligent \& Fuzzy Systems, vol. 29, no. 5, pp. 2227-2233, 2015.

[46] M. Tutuianu, A. Marotta, H. Steven et al., Development of a World-Wide Worldwide Harmonized Light Duty Driving Test Cycle (WLTC), UNECE, Geneva, Swizerland, 2014.

[47] D. J. Cole, "A path-following driver-vehicle model with neuromuscular dynamics, including measured and simulated responses to a step in steering angle overlay," Vehicle System Dynamics, vol. 50, no. 4, pp. 573-596, 2012.

[48] E. Tzirakis and F. Zannikos, "Impact of driving styles on fuel consumption and exhaust emissions: defensive and aggressive driving style," in Proceedings of the 10th International Conference on Environmental Science and Technology, pp. 1497-1504, Cos island, Greece, September 2007.

[49] Y. L. Murphey, R. Milton, and L. Kiliaris, "Driver's style classification using jerk analysis," in Proceedings of the 2009 IEEE Workshop on Computational Intelligence in Vehicles and Vehicular Systems, pp. 23-28, Nashville, TN, USA, April 2009.

[50] G. F. Li, Y. F. Yang, T. R. Zhang et al., "Risk assessment based collision avoidance decision-making for autonomous vehicles in multi-scenarios," Transportation Research Part C: Emerging Technologies, vol. 122, Article ID 102820, 2021. 